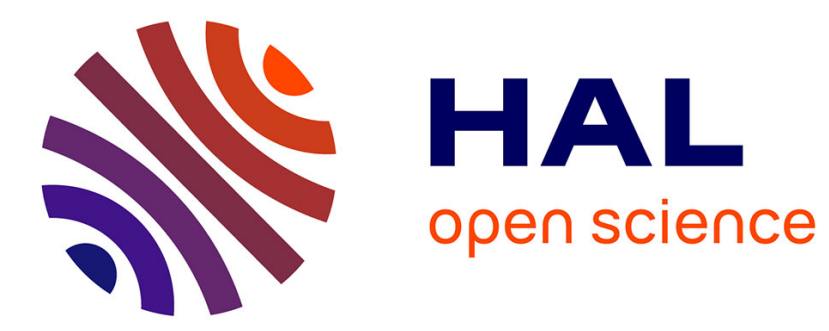

\title{
On the Use of Wide Channels in WiFi Networks
}

\author{
Saber Malekmohammadi, Catherine Rosenberg, Razvan Stanica
}

\section{To cite this version:}

Saber Malekmohammadi, Catherine Rosenberg, Razvan Stanica. On the Use of Wide Channels in WiFi Networks. LCN 2019 - 44th IEEE Conference on Local Computer Networks, Oct 2019, Osnabruck, Germany. pp.1-4. hal-02336041

\section{HAL Id: hal-02336041 \\ https://hal.inria.fr/hal-02336041}

Submitted on 28 Oct 2019

HAL is a multi-disciplinary open access archive for the deposit and dissemination of scientific research documents, whether they are published or not. The documents may come from teaching and research institutions in France or abroad, or from public or private research centers.
L'archive ouverte pluridisciplinaire HAL, est destinée au dépôt et à la diffusion de documents scientifiques de niveau recherche, publiés ou non, émanant des établissements d'enseignement et de recherche français ou étrangers, des laboratoires publics ou privés. 


\title{
On the Use of Wide Channels in WiFi Networks
}

\author{
Saber Malekmohammadi*, Catherine Rosenberg*, Razvan Stanica ${ }^{\dagger}$ \\ * Department of Electrical and Computer Engineering, University of Waterloo, Canada \\ ${ }^{\dagger}$ Univ Lyon, INSA Lyon, Inria, CITI, F-69621 Villeurbanne, France
}

\begin{abstract}
An increased density of access points is common today in WiFi deployments, and more and more parameters need to be configured in such networks. In this paper, we question current industrial guidelines for both residential and enterprise scenarios. More precisely, we investigate the joint channel, power, and carrier sense threshold allocation problem in IEEE 802.11ac networks, showing that the current practice, which is to use narrower channels at maximum power when the deployment is dense, yields much worse performance than a solution using the widest possible channel with a much lower power.
\end{abstract}

Index Terms-WiFi, Channel Allocation, Power Management

\section{INTRODUCTION}

Classically, deploying a WiFi network in the $2.4 \mathrm{GHz}$ band consists in choosing the best $20 \mathrm{MHz}$ channel for each access point (AP) from an interference point of view. However, this problem drastically changes with the evolution of the technology. Nowadays, the administrator of an IEEE 802.11ac wireless local area network (WLAN) has to select not only the channel width for each AP, but also the specific channel to use when several possibilities exist for a given width. The transmission power and the the carrier sense threshold (CST) of each AP can be modified, resulting is a complex joint channel, power and CST allocation problem.

In this paper, we explore whether it is better, in terms of overall performance for each AP, to use wide channels, in spite of the increased interference and collision region which appear in this case, or narrow channels in order to reduce interference and decrease the collision region. The widely accepted approach in the literature on WLAN configuration [1][2][3] is to allocate narrower channels and lower AP transmission power as the network density (in terms of APs) increases. Moreover, in residential WiFi networks, where the deployment and configuration of the APs is not coordinated, the default practice is to use narrow $20 \mathrm{MHz}$ channels and the maximum authorised transmission power. In our work, we question these approaches and study the interplay between channel bandwidth, transmission power and the value of CST on the performance of the network.

Our contributions can be summarized as follows. We consider a WLAN operating on a single $160 \mathrm{MHz}$ band, which can be divided in 8/4/2/1 non-overlapping 20/40/80/160 MHz channels. Through an extensive simulation campaign, we focus on a scenario with densely deployed APs, each serving a relatively low number of users with important downlink traffic. In these conditions, we first show that the current default settings for residential APs are underperforming. Indeed, our results show that the performance obtained on the single 160
$\mathrm{MHz}$ channel with low transmission power is significantly higher than the one observed when using the maximum transmission power, irrespective of the channel allocation. We also notice that, in such dense deployments, some APs see degraded performance, depending on the WLAN topology. Our tests show that, by using higher transmission power and CST for the poor APs, these APs can be brought up to level.

\section{RELATED WORK}

Channel allocation, power management and CST adjustment have been studied in centrally coordinated WiFi networks. In [4] and [5], channel assignment and AP placement problems were investigated, assuming the same fixed width for all the channels and fixed transmission power and CST for all APs. A channel allocation heuristic considering the traffic load at the MAC layer is formulated in [6]. However, the study assumes fixed channel bandwidth, transmission power and CST value. A Dynamic Sensitivity Control (DSC) algorithm is proposed in the IEEE 802.11ax amendment, and [7] shows that, by dynamically adjusting the CST threshold in a dense network, its aggregate throughput can be considerably improved. Again, it is assumed that the channels have a fixed bandwidth and transmission power values are fixed.

The most complete solutions in the area of WLAN management come from Cisco Systems: a centrally controlled power allocation algorithm (TPC) [8], and a dynamic channel assignment (DCA) algorithm [2]. In DCA, the central controller gathers information from all the APs in the network and calculates a goodness metric for each channel and for each AP, based on the received signal strength indicator (RSSI). DCA finds the AP with the lowest current goodness and tries to improve it, by assigning it a different, more suitable channel. This process is performed iteratively by the controller, but the general principle remains to assign narrower channels to APs in the network as the deployment density increases.

The TPC algorithm assigns (possibly different) transmission power values $\left(P_{t}\right)$ to APs, aiming to maximize their coverage area and minimize their interference potential. Like DCA, TPC runs in a central controller, and its objective is to assign to each AP a transmission power $T x_{i d e a l}$, computed as follows:

$$
T x_{i d e a l}(i)=T x_{\max }-\left(R S S I_{3 r d}(i)-T P C_{\text {threshold }}\right),
$$

where $T x_{\max }$ is the maximum power allowed by regulators, $T P C_{\text {threshold }}$ is a customizable power threshold, and $R S S I_{3 r d}(i)$ is the RSSI of the third largest interfering AP in the network, as measured by AP $i$. 
DCA and TPC are executed by a central controller but independently from each other [9]. Other WiFi actors [1] use a similar method for channel allocation and power management. In summary, most of the works in the literature study the effect of only one of the channel bandwidth, transmission power and CST parameters on WLAN performance, and, to the best of our knowledge, there is no prior work that studies the interplay of the three parameters together.

\section{BENCHMARKS}

Our main question in this work can be formulated as follows: given an already deployed WiFi network, what is the best combination of channel bandwidth $\left(W_{j}\right)$, channel identity, total transmission power $\left(P_{j}\right)$ and channel sensing threshold $\left(C S T_{j}\right)$ to allocate to AP $j$ ? We begin by discussing two state of the art channel allocation and power management schemes for enterprise WLANs and we take them as benchmarks.

1) Benchmark 1 - Using narrower channels as the deployment density increases: One common approach ([1], [2], [3]) is to assign narrower channels to APs in dense deployments. This way, APs get less bandwidth, but also less contention. Assuming that all APs use the same channel width, the same CST and their maximum power budget, the only two parameters we need to determine are the value $W$ of the channel width and the frequency reuse pattern. The works above show that, under these assumptions, it is better to use $W=20 \mathrm{MHz}$ when the network is dense, while $W$ can be larger for sparser networks. Note that none of these studies proposes a clear criteria to define a dense network in terms of AP. This approach is representative of a residential WLAN scenario, where an AP usually uses default parameters or a dynamic approach based on local measurements.

2) Benchmark 2 - Decoupled channel allocation and power control: Some studies [9] simplify the $\left(W_{j}, P_{j}, C S T_{j}\right)$ allocation problem by assuming the same fixed CST for all APs and by performing a dynamic channel assignment (e.g. DCA [2]) and a transmit power control (e.g. TPC [8]), separately from each other. The two algorithms are performed sequentially by a central controller connected to all APs, but it is obvious that changes in power allocation brought by TPC will yield changes in the results of DCA and vice-versa. While TPC allocates different powers to different APs, we can compute a lower bound on all the allocated powers by using the lowest possible value for $T P C_{\text {threshold }}(-80 \mathrm{dBm}$ in [8]). In the following, we use as a second benchmark the combination of static DCA along with this lower bound provided by TPC. This approach is representative of enterprise and campus WiFi networks, where such a centralization is possible.

\section{NeTwork Model}

We use the ns-3 simulator for studying realistic WiFi networks of different densities. For simplicity, we only consider downlink transmissions in this work. However, we note that this does not imply a zero uplink traffic, since the STAs transmit an acknowledgement message to the corresponding

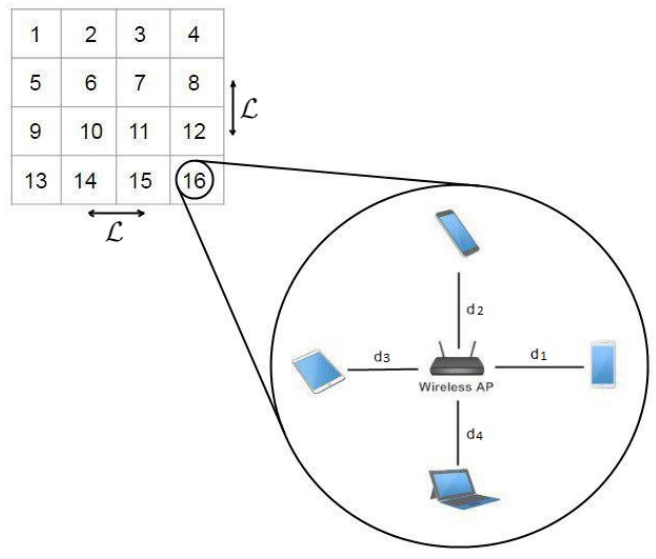

Fig. 1. One generic floor of the simulated network (four floors overall).

AP for every frame received correctly. We computed all simulation results with $99 \%$ confidence intervals but, since the obtained intervals are very tight, we do not show them in the figures, for better readability.

We consider WiFi networks consisting of 64 identical APs located on four floors of a building. On each floor, there are 16 APs, each of which is located in one square room, as shown in Figure 1. APs and users in each floor are mounted $1 \mathrm{~m}$ above that floor. The AP in each room is located randomly in a square of length $5 \mathrm{~m}$, concentric with the room. The horizontal distance between two adjacent floors is set to $4 \mathrm{~m}$. The distance between the centers of adjacent rooms (located on the same floor) is denoted as $\mathcal{L}$. Therefore, the density of the system in terms of AP can be changed by changing the value of $\mathcal{L}$, which allows us to easily switch between sparser (e.g. residential) and denser (e.g. enterprise) scenarios. There are 4 users located inside each room, associated to the AP in the same room (see Figure 1) and placed randomly at a distance up to $\mathcal{L} / 2(\mathrm{~m})$ from the AP.

We assume a full buffer UDP traffic model with a uniform arrival rate equal to $230 \mathrm{Mbps}$; i.e., the APs always have data to transmit to their users. The overall performance metric that we use is the geometric mean (GM) of the throughput of all the users existing in the network. The higher the GM, the more efficient and fair is the network [10].

We use the ns-3 Log Distance Propagation Loss Model and add wall penetration loss effects on top of that by using the ns-3 Building Class. Between two adjacent rooms, there is a wall penetration loss equal to $8 \mathrm{~dB}$. The APs antenna gain is equal to $12 \mathrm{dBi}$. The antenna gain on the user side is equal to $0 \mathrm{dBi}$ and the number of spatial streams used by the APs is set to one. Whenever channel bonding is used by the IEEE 802.11ac APs, we assign them the same primary channel.

Regarding the transmission power, every country imposes a limit for the total RF power that can be radiated by antennas in the subband that is used. In this study, we consider a maximum power of $23 \mathrm{dBm}(200 \mathrm{~mW})$. Therefore, when using a channel with bandwidth $20,40,80$ and $160 \mathrm{MHz}$, the maximum power 


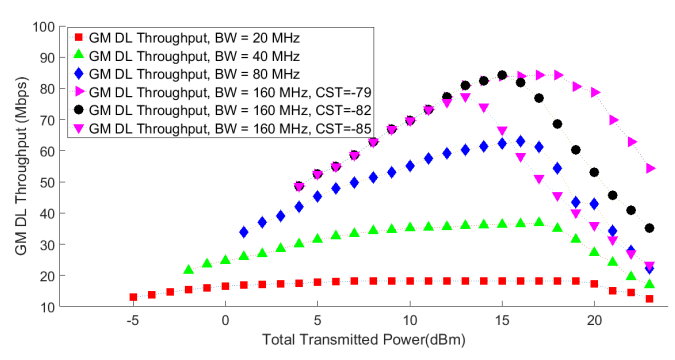

Fig. 2. The network overall performance vs. $P_{t}$ for different channel widths and different CSTs, for $\mathcal{L}=40 \mathrm{~m}$.

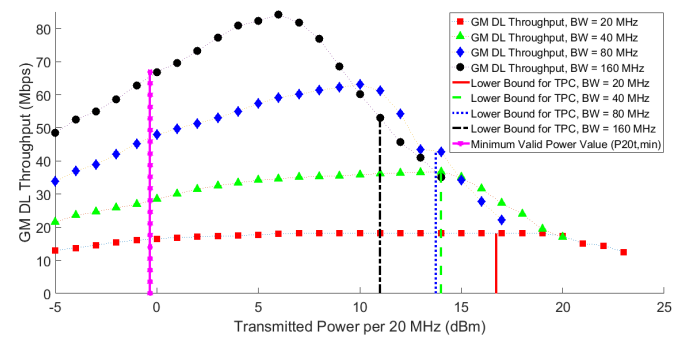

Fig. 3. The network overall performance vs. $P_{t}^{20}$ for different channel widths for the default CST, for $\mathcal{L}=40 \mathrm{~m}$.

that can be transmitted over each $20 \mathrm{MHz}$ subchannel, denoted as $P_{t}^{20}$, is equal to $23,20,17$ and $14 \mathrm{dBm}$, respectively. A minimum transmission power, denoted as $P_{t, \text { min }}^{20}$, can also be computed, considering that each AP needs to have a coverage range which is at least equal to the distance to the farthest corner of its room. Therefore, given a channel of a specific width allocated to an AP, the values between the computed $P_{t, \text { min }}^{20}$ and the maximum $P_{t}^{20}$ allowed by local regulations represent the valid range of power values for a given AP.

\section{UNIFORM AP SETTINGS}

We first allocate the same channel bandwidth, the same $P_{t}^{20}$ value and the same CST to all the APs in our simulated network, not unlike a residential deployment of APs with default settings. We consider the case where $\mathcal{L}=40 \mathrm{~m}$, which corresponds to a network of low AP density. In Figure 2, we show the results as a function of the total $P_{t}$ for different CSTs, while in Figure 3 we show the same results as a function of $P_{t}^{20}$, but for only the default CST of $-82 \mathrm{dBm}$.

The first comment based on Figure 2 is that using the widest channel $(160 \mathrm{MHz})$ provides the highest overall performance. The second comment is that the peak performance is obtained for a total transmit power of $15 \mathrm{dBm}$. As for the CST parameter, it only shows an impact when the APs use a high transmission power, mainly because, at low power values, the APs sense each other transmissions much less. Consequently, decreasing or increasing their CST does not affect their throughput much.

The best observed performancen in terms of GM is around $84 \mathrm{Mbps}$ and is obtained for $W=160 \mathrm{MHz}$ and for a relatively low power, i.e., $P_{t}^{20} \approx 6 \mathrm{dBm}$ (which is in the valid range of powers). Note that the lower channel widths give much lower peaks. Benchmark 1 would use the maximum power $P_{t}$ and then would also select $W=160 \mathrm{MHz}$, resulting in a performance of only $35 \mathrm{Mbps}$. To compare with Benchmark 2, we show five vertical lines in Figure 3. The leftmost one shows the minimum power value necessary for coverage, i.e., $P_{t, \min }^{20}$. The four vertical lines at the right of the figure show the lower bounds for the output values of the TPC algorithm for each channel width. The best possible values that could be selected by Benchmark 2 are $W=160$ $\mathrm{MHz}$ and $P_{t}^{20}=11 \mathrm{dBm}$, yielding an overall performance of 53 Mbps, better than Benchmark 1 but still 58\% lower than the peak we observe.

We then increase the density of the network to $\mathcal{L}=15 \mathrm{~m}$ and we report the results in terms of $P_{t}^{20}$ in Figure 4, when CST has the default value. As in the previous case, we show the five vertical lines depicting the performance of the two benchmark solutions.

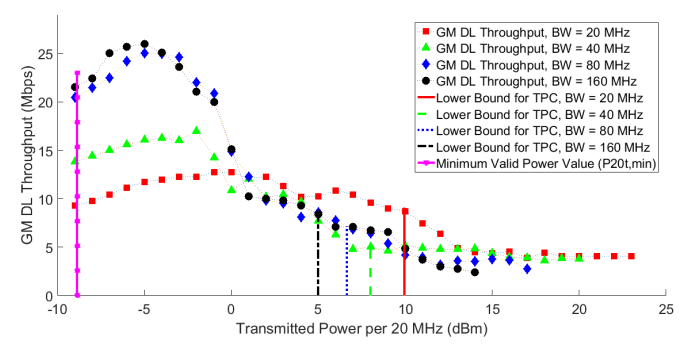

Fig. 4. The network overall performance vs. $P_{t}^{20}$ for different channel widths, for $\mathcal{L}=15 \mathrm{~m}$.

The best performance is $\approx 26 \mathrm{Mbps}$ and is obtained for $W=160 \mathrm{MHz}$ (though $W=80 \mathrm{MHz}$ does almost as well in this case) and for a low power, i.e., $P_{t}^{20} \approx-5 \mathrm{dBm}$. Benchmark 1 would use the maximum power $P_{t}$ and then would select $W=20 \mathrm{MHz}$, with a performance of around $5 \mathrm{Mbps}$, about one fifth of the best achievable performance. To compare with Benchmark 2, we again look at the four rightmost vertical lines in Figure 4. The best possible values that could be selected by Benchmark 2 are $W=20 \mathrm{MHz}$ and $P_{t}^{20}=10 \mathrm{dBm}$ (a relatively high power), yielding an overall performance of about $9 \mathrm{Mbps}$.

To conclude this part, in our scenarios, using a wide channel with low power is significantly more effective than using a narrow channel with any power, irrespective of the density of the network. This challenges the current default settings in residential WiFi deployment, which follow settings similar to Benchmark 1.

\section{NON-UNIFORM AP SETTINGS}

While the results shown in the previous section demonstrate impressive gains in terms of GM throughput, in this section we further explore the distribution of performance among the APs. More precisely, when using the widest available channel $(160 \mathrm{MHz})$, all the APs in the network share the 
same resources, suffering from co-channel interference and cochannel contention. So far, we tried to mitigate the interference and reduce the contention by adjusting power, while keeping the same CST for all APs, achieving a significant improvement in the network overall performance. Going a step further, we show in Figure 5 the GM of the throughput obtained on each of the 64 APs (four users associated to each AP), when $\mathcal{L}=$ $15 \mathrm{~m}$. In these results, the APs are all allocated the widest channel $(160 \mathrm{MHz})$, use the corresponding best power value (corresponding to the peak point in the previous section) and the default CST value.

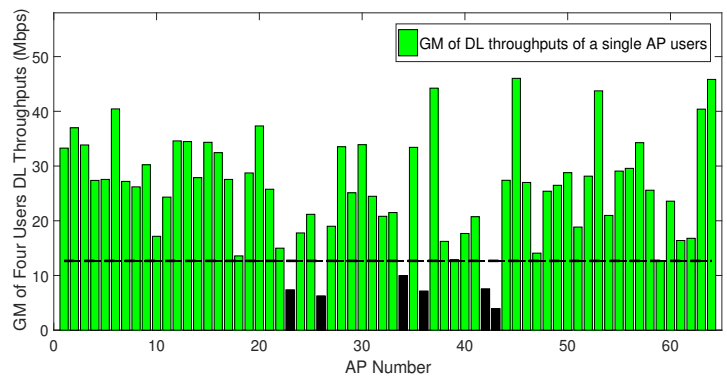

Fig. 5. The GM throughput of the 64 APs in the network for the power yielding the best overall GM and $\mathcal{L}=15 \mathrm{~m}$. The dashed line represents half of the arithmetic mean of the GM throughput of the APs.

As it can be seen in Figure 5, some APs see much lower performance than others: APs 23, 26, 34, 36, 42 and 43, i.e., those located in the middle of the network. Specifically, we say that an AP is poor if it receives less than half of the arithmetic mean of the GMs of all APs (the dashed line in Figure 5 shows this value). The reasons why the APs located in the middle of the enterprise network are poor are twofold. The first one is that these APs have to contend with a lot of surrounding APs, while this is not the case for APs at the edges. Therefore, one suggestion for helping the poor APs is to increase their CST so that they can get access to the shared channel more easily. The second reason is the higher amount of co-channel interference that the users of these APs receive. Hence, one other suggestion for improving the performance of these APs is to increase their transmission power values slightly (the other APs keep the power value determined for the case with equal parameters) so that they can combat the higher interference that they experience.

To test these ideas, we consider two values for the CST of the poor APs: $-79 \mathrm{dBm}$ and $-76 \mathrm{dBm}$ (recall that the default value of the CST used so far was $-82 \mathrm{dBm}$ ). We also increase their transmission power value by $3 \mathrm{dBm}$ and $6 \mathrm{dBm}$ with respect to rich APs, which are allocated their peak point power value. We consider all the possible combinations for changing the CST and power value of the poor APs and report these results in Table I. The table contains the overall GM throughput $G M_{t o t}$, the overall arithmetic mean throughput, $A M_{t o t}$, and the minimum of the GM throughput of each AP.

Table I shows that, although we only slightly increase the overall GM (about 6\%), adjusting the CST and the TX power
TABLE I

PERFORMANCE UNDER NON-UNIFORM AP SETTINGS WHEN USING OPTIMAL SETTINGS. $\mathrm{C}_{\text {poor }}+$ (RESP. $\mathrm{P}_{\text {poor }}$ ) IS THE INCREMENT OF CST (RESP. TX POWER) FOR POOR USERS.

\begin{tabular}{|c|c|c|c|c|}
\hline $\mathrm{C}_{\text {poor }}+$ & $\mathrm{P}_{\text {poor }}+$ & $G M_{\text {tot }}$ & $A M_{\text {tot }}$ & $G M_{\min }$ \\
\hline none & none & 22.8 & 29.51 & 3.92 \\
\hline 3 & none & 23.18 & 29.72 & 9.23 \\
6 & none & $\mathbf{2 4 . 2 4}$ & $\mathbf{3 0 . 0 5}$ & $\mathbf{9 . 4 7}$ \\
\hline none & 3 & 22.27 & 29.14 & 7.21 \\
\hline 3 & 3 & 21.04 & 28.58 & 7.49 \\
3 & 6 & 21.14 & 28.56 & 7.52 \\
\hline
\end{tabular}

for the poor APs significantly increase the minimum GM throughput. Therefore, our results show that, in a centralized WLAN, unfairness among APs can be mitigated by fine tuning the CST and TX power of the APs with poor performance.

\section{CONCLUSION}

With the increased traffic demand, WLANs become denser and denser in terms of AP deployment. This raises new challenges in terms of resource allocation and several elements remain unaccounted for in the state of the art. In this context, we studied the channel allocation, power management and CST adjustment problems jointly in IEEE 802.11ac networks. We observed that, when allocating channels in a densely deployed WiFi network, using the maximum allowed transmission power by APs is not appropriate. This questions the current approach in residential WiFi networks, where default settings with high transmission powers are used. Our results also show that using homogeneous CST and power settings in a network yields unfairness among APs. We observe that modifying the CST of poor APs alleviates this unfairness problem and brings their performance up to level.

\section{REFERENCES}

[1] Cisco Meraki, High Density Wi-Fi Deployments Guide, [Online]. Available: https://meraki.cisco.com/lib/pdf/meraki_high-density_ solution_guide.pdf.

[2] Cisco Systems, Dynamic Channel Assignment (DCA), [Online]. Available: https://www.cisco.com/c/en/us/td/docs/wireless/controller/ technotes/8-3/b_RRM_White_Paper/b_RRM_White_Paper_chapter_ 0100.pdf.

[3] Aruba Networks, Very High-Density 802.11ac Networks Planning Guide., [Online]. Available: https://www.johnlose.de/wp-content/ uploads/2018/07/Aruba_VHD_VRD_Planning_Guide.pdf.

[4] A. Hills, "Large-Scale Wireless LAN Design," IEEE Communications Magazine, vol. 39, no. 11, pp. 98-107, Nov. 2001.

[5] R. C. Rodrigues, G. R. Mateus, and A. A. F. Loureiro, "On the Design and Capacity Planning of a Wireless Local Area Network," in Proc. IEEE/IFIP NOMS, Apr. 2000.

[6] K. K. Leung and B. J. Kim, "Frequency Assignment for IEEE 802.11 Wireless Networks," in Proc. IEEE VTC Fall, Oct. 2003.

[7] M. S. Afaqui, E. Garcia-Villegas, E. Lopez-Aguilera, G. Smith, and D. Camps, "Evaluation of Dynamic Sensitivity Control Algorithm for IEEE 802.11ax," in Proc. IEEE WCNC, Mar. 2015.

[8] Cisco Systems, Transmit Power Control (TPC), [Online]. Available: https://www.cisco.com/c/en/us/td/docs/wireless/controller/technotes/8-3/ b_RRM_White_Paper/b_RRM_White_Paper_chapter_0101.html.

[9] —-, Radio Resource Management White Paper., [Online]. Available: https://www.cisco.com/c/en/us/td/docs/wireless/controller/technotes/8-3/ b_RRM_White_Paper.html.

[10] D. Fooladivanda and C. Rosenberg, "Joint Resource Allocation and User Association for Heterogeneous Wireless Cellular Networks," IEEE Transactions on Wireless Communications, vol. 12, no. 1, Jan. 2013. 